\title{
Diosgenin prevents high-fat diet-induced rat non-alcoholic fatty liver disease through the AMPK and LXR signaling pathways
}

\author{
SILU CHENG ${ }^{1 *}$, SHUFANG LIANG $^{1 *}$, QUN LIU $^{1 *}$, ZHENGTING DENG $^{1,2}$, YUANHUI ZHANG $^{1}$, \\ JUAN DU ${ }^{1}$, YA'NI ZHANG ${ }^{1},{\text { SHU } L^{3}}^{3}$, BINBIN CHENG ${ }^{1}$ and $\mathrm{CHANGQUAN} \mathrm{LING}^{1,4}$ \\ ${ }^{1}$ Department of Traditional Chinese Medicine, Changhai Hospital, The Second Military Medical University, Shanghai 200433; \\ ${ }^{2}$ Department of Oncology, Suzhou Hospital of Traditional Chinese Medicine, Suzhou, Jiangsu 215009; \\ ${ }^{3}$ Department of Gastroenterology, Baoshan Branch, Shuguang Hospital Affiliated to Shanghai University \\ of Traditional Chinese Medicine, Shanghai 201900; ${ }^{4}$ E-Institute of TCM Internal Medicine, \\ Shanghai Municipal Education Commission, Shanghai 201203, P.R. China
}

Received March 13, 2017; Accepted November 9, 2017

DOI: $10.3892 /$ ijmm.2017.3291

\begin{abstract}
Non-alcoholic fatty liver disease (NAFLD) is a major public health concern worldwide. The aim of the present study was to observe the effect of diosgenin on NAFLD and investigate the underlying mechanisms. Diosgenin treatment increased the phosphorylation of AMP-activated protein kinase (AMPK) and acetyl-CoA carboxylase (ACC) in HepG2 cells. Diosgenin significantly inhibited high glucose (HG)-induced triglyceride (TG) accumulation and sterol regulatory element-binding protein-1c (SREBP-1c) mRNA increase in HepG2 cells, which were partially abolished by the AMPK inhibitor compound C. Diosgenin also significantly inhibited the increase of liver $\mathrm{X}$ receptor (LXR) $\alpha$ mRNA induced by HG or T0901317. However, T0901317-induced upregulation of LXR $\alpha$ and SREBP-1c mRNA was not blocked by compound C. Following a high-fat diet for 16 weeks, the body and liver weights of the experimental rats were significantly increased, but this effect was significantly suppressed by diosgenin. Diosgenin and fenofibrate ameliorated lipid deposition in the liver and reduced the increase of hepatic TG content. Diosgenin significantly decreased the alanine aminotransferase (ALT) level, whereas fenofibrate significantly increased the ALT and aspartate aminotransferase levels. Diosgenin also increased AMPK and ACC phosphorylation and suppressed LXR $\alpha$ in the liver. In conclusion, the results of the present study suggested that
\end{abstract}

Correspondence to: Dr Binbin Cheng or Dr Changquan Ling, Department of Traditional Chinese Medicine, Changhai Hospital, The Second Military Medical University, 168 Changhai Road, Shanghai 200433, P.R. China

E-mail: cbb8202@126.com

E-mail: changquanling@smmu.edu.cn

*Contributed equally

Key words: AMP-activated protein kinase, diosgenin, high glucose, insulin resistance, non-alcoholic fatty liver disease diosgenin is a potential agent for preventing the development of NAFLD through the AMPK and LXR signaling pathways.

\section{Introduction}

Non-alcoholic fatty liver disease (NAFLD), which is characterized by excessive triglyceride (TG) accumulation in hepatocytes, has become a major public health concern worldwide, with an estimated prevalence range of $24-42 \%$ in Western countries and 5-40\% in Asian countries (1,2). NAFLD may progress to steatohepatitis and fibrosis, or even cirrhosis, liver cancer and liver failure over time (3). The mechanisms underlying the development of NAFLD have not yet been fully elucidated. Since obesity and diabetes are considered to be major risk factors for the development and progression of NAFLD, current therapies for NAFLD are mainly aimed at reducing body weight and improving insulin sensitivity (4).

AMP-activated protein kinase (AMPK) plays an important role in regulating hepatic lipogenesis (5). In the liver, activation of AMPK by phosphorylation of threonine 172 induces the phosphorylation and inactivation of acetyl-CoA carboxylase (ACC), subsequently leading to the suppression of fatty acid synthesis (6). In addition, AMPK is a negative sterol regulatory element-binding protein-1c (SREBP-1c), a key regulator of TG metabolism (7). Phosphorylation of AMPK downregulates the activity of SREBP-1c $(8,9)$. However, the activity of AMPK is dysregulated in patients with metabolic syndromes, such as diabetes and obesity $(10,11)$. Thus, stimulation of AMPK activation, which thereby suppresses ACC and SREBP-1c activity, may alleviate hepatic accumulation of TG.

Our institute has expressed a continuous interest in the prevention and treatment of chronic liver diseases with traditional Chinese medicine. Diosgenin, which is abundant in Chinese yam (Dioscorea villosa) and Rhizoma Dioscoreae Nipponicae, has been shown to lower increased plasma glucose levels and improve the distorted tissue lipid profile in high-fat diet (HFD)-streptozotocin-induced diabetic rats (12). Furthermore, diosgenin was able to induce AMPK phosphorylation under basal as well as inflammatory conditions in perivascular adipose tissue (13). These results 
indicate that diosgenin may be an effective treatment for NAFLD. Therefore, the effect of diosgenin on HFD-induced rat NAFLD was observed, and the underlying mechanisms were investigated in the present study.

\section{Materials and methods}

Cell culture. The human liver cancer HepG2 cell line was obtained from the CellBank of Chinese Academy of Sciences and cultured in Dulbecco's modified Eagle's medium (DMEM) containing normal glucose [5.5 mM, D-glucose; low glucose (LG)] supplemented with $10 \%$ fetal bovine serum (FBS), $100 \mathrm{U} / \mathrm{ml}$ penicillin and $0.1 \mathrm{mg} / \mathrm{ml}$ streptomycin at $37^{\circ} \mathrm{C}$ in a humidified atmosphere of $5 \% \mathrm{CO}_{2}$. To examine the accumulation of TG, HepG2 cells were maintained in serum-free DMEM overnight, as previously described (14), and the cells were then treated with indicated concentrations of diosgenin in DMEM containing a high concentration of glucose $[30 \mathrm{mmol} / \mathrm{l}$; high glucose $(\mathrm{HG})]$ for another $24 \mathrm{~h}$, as previously described $(14,15)$. The cells were then lysed and TG levels were determined using a commercial kit (Nanjing Jiancheng Bioengineering Institute, Nanjing, China) according to the manufacturer's instructions.

Reverse transcription-quantitative polymerase chain reaction $(P C R)$. Total RNA was extracted from HepG2 cells after diosgenin treatment with TRIzol reagent (Invitrogen Life Technologies; Thermo Fisher Scientific, Inc., Carlsbad, CA, USA) according to the manufacturer's instructions. Total RNA (500 ng) was reverse-transcribed into cDNA using a First-Strand cDNA synthesis kit (FSK-100; Toyobo, Osaka, Japan). The amplification of the cDNA was accomplished in triplicate using SYBR-Green PCR Master Mix (Toyobo). The cDNA was amplified under the following conditions: $95^{\circ} \mathrm{C}$ for 5 min for denaturation, followed by 40 cycles at $95^{\circ} \mathrm{C}$ for $10 \mathrm{sec}, 60^{\circ} \mathrm{C}$ for $20 \mathrm{sec}$ and $72^{\circ} \mathrm{C}$ for $25 \mathrm{sec}$. The primers used in the present study were as follows: SREBP-1c forward, 5'-ACCGACATCGAAGGTGAAGT-3' and reverse, CCAGCA TAGGGTGGGTCAAA; LXR $\alpha$ forward, 5'-GGACCAGCT CCAGGTAGAGA-3' and reverse, 5'-ACACTTGCTCTGAGT GGACG-3'; and $\beta$-actin forward, 5'-AGCGGGAAATCGTGC GTG-3' and reverse, 5'-CAGGGTACATGGTGGTGCC-3'. The relative expression level of mRNA in each sample was normalized to its $\beta$-actin content. The relative expression levels of mRNA were calculated with the $2^{-\Delta \Delta \mathrm{Cq}}$ method.

Western blot analysis. Total protein was extracted as previously described (16). The protein concentration was determined by the bicinchoninic acid method. Equal quantities of proteins were separated by sodium dodecyl sulfate-polyacrylamide gel electrophoresis and transferred by electroblotting to a nitrocellulose membrane. The membrane was blocked with 5\% BSA in TBST buffer (20 mM Tris-HCl, pH 7.4, $150 \mathrm{mM}$ $\mathrm{NaCl}$ and $0.1 \%$ Tween-20) overnight at $4^{\circ} \mathrm{C}$. Subsequently, the membrane was incubated with specific primary antibodies [rabbit monoclonal antibodies (1:1,000) AMPK (no. 2603), p-AMPK (no. 2535), ACC (no. 3676), and rabbit polyclonal antibody $(1: 1,000)$ pACC (no. 3661) (all from Cell Signaling Technology, Inc., Danvers, MA, USA), goat polyclonal antibody LXR $\alpha$ (1:200; sc-1202; Santa Cruz Biotechnology, Inc., Santa Cruz, CA, USA), rabbit monoclonal antibody $\beta$-actin (1:5,000; no. 4970; Cell Signaling Technology, Inc.)] overnight at $4^{\circ} \mathrm{C}$, followed by incubation with a secondary antibody [anti-rabbit IgG (no. 7074; Cell Signaling Technology, Inc.) or anti-goat IgG (sc-2354; Santa Cruz Biotechnology, Inc.)] for $1 \mathrm{~h}$. The signal was visualized with an enhanced chemiluminescence (ECL) kit (Thermo Fisher Scientific, Inc., Carlsbad, CA, USA).

Animals. A total of 40 male Sprague-Dawley rats (6-8 weeks old, weighing $250 \pm 20 \mathrm{~g}$ ) were purchased from the Shanghai Laboratory Animal Center and kept at the Second Military MedicalUniversity Laboratory AnimalCenter.The animals were maintained on a 12-h light/dark cycle (7:00 a.m.-19:00 p.m.), at $22 \pm 2^{\circ} \mathrm{C}$, with food and water available ad libitum. To avoid the effect of environmental changes, all the animals were housed for 1 week in the controlled environment prior to use in the experiment. All the procedures were performed in accordance with the institutional guidelines for animal research. The present study was approved by the Committee on Ethics of Biomedicine, the Second Military Medical University.

Experiment design and drug administration. The animals were randomly assigned into 5 groups $(n=8)$ : Control group (C), model group (M), high-dose diosgenin group (HDD), low-dose diosgenin group (LDD) and fenofibrate group (FEN). The rats in the model, HDD, LDD and FEN groups were fed HFD, HFD mixed with $1 \%$ (wt/wt) diosgenin, HFD mixed with $0.5 \%$ (wt/wt) diosgenin and HFD mixed with $0.02 \%$ fenofibrate, respectively. The control group was given the same amount of food as the other groups. HFD was provided by the Shanghai Laboratory Animal Center. The energy composition of the HFD consists of $45 \%$ fat, $18 \%$ protein and $37 \%$ carbohydrate. A regular rat diet (10\% fat, $22 \%$ protein, $68 \%$ carbohydrate) was used as the maintenance and control diet.

After 16 weeks of feeding according to previous studies $(17,18)$, blood was collected from the retro-orbital venous plexus for detecting the content of serum total cholesterol (TC) and TG and the liver function. The rat liver was removed and frozen in liquid nitrogen for the following experiments.

Detection of serum aspartate aminotransferase (AST) and alanine aminotransferase (ALT) activity. Serum ALT and AST were determined using biochemical kits (Nanjing Jiancheng Bioengineering Institute) according to the manufacturer's instructions.

Hematoxylin and eosin $(H \& E)$ and Oil red $O$ staining. Liver tissue was embedded in Tissue-Tek optimum cutting temperature compound (Sakura Finetek, Torrance, CA, USA), quickly frozen by immersion in liquid nitrogen, and stained with H\&E. Alternatively, intrahepatic lipids were stained by the Oil red O method, as previously described (19). Images were obtained using a Leica inverted fluorescence microscope (Leica Microsystems GmbH, Wetzlar, Germany).

Statistical analysis. Data are expressed as means \pm standard deviation. One-way analysis of variance followed by Student-Newman-Keuls tests were used for statistical analysis. Statistical significance was established at $\mathrm{P}<0.05$. 
A

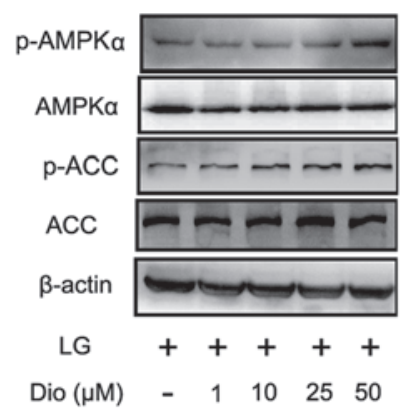

D

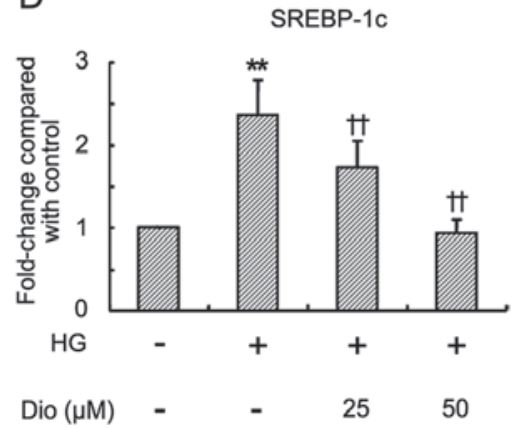

B

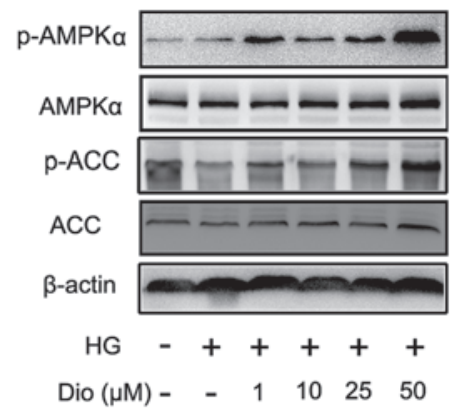

E

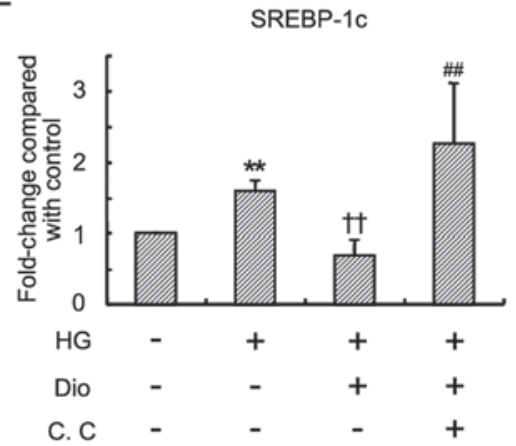

C

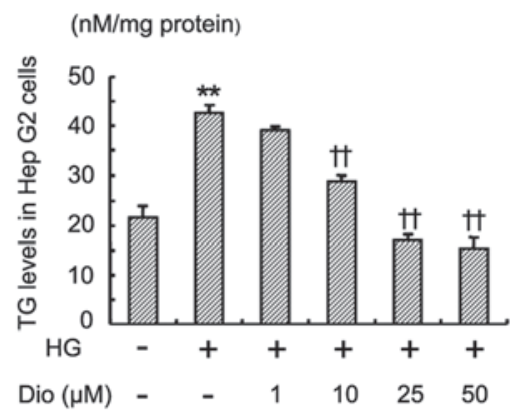

F

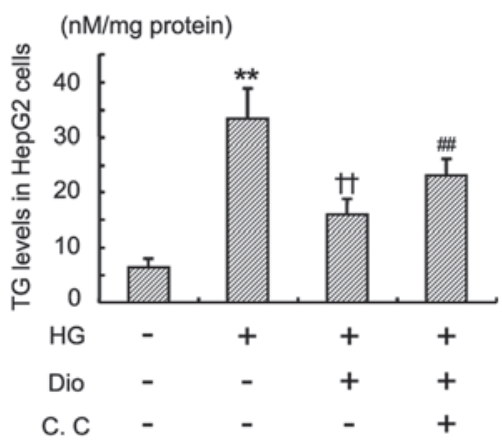

Figure 1. Diosgenin (Dio) increases AMP-activated protein kinase (AMPK) and acetyl-CoA carboxylase (ACC) phosphorylation and suppresses high glucose (HG)-induced triglyceride (TG) accumulation in HepG2 cells. (A) Diosgenin increases AMPK and ACC phosphorylation under low glucose (LG) conditions; (B) diosgenin increases AMPK and ACC phosphorylation under HG conditions; (C) diosgenin inhibits HG-induced TG accumulation in HepG2 cells; (D) diosgenin inhibits HG-induced increase of sterol regulatory element-binding protein-1c (SREBP-1c) mRNA in HepG2 cells; (E and F) compound C (C.C) blocks the effect of diosgenin on SREBP-1c mRNA expression and TG accumulation in HepG2 cells. The data are presented as mean \pm standard deviation, $\mathrm{n}=3$. ${ }^{* *} \mathrm{P}<0.01$ compared with the control group; ${ }^{\dagger \dagger} \mathrm{P}<0.01$ compared with the HG group; ${ }^{\# \#} \mathrm{P}<0.01$ compared with the diosgenin group.

\section{Results}

Diosgenin increases AMPK and ACC phosphorylation and suppresses $H G$-induced TG accumulation in HepG2 cells. In view of the important role of AMPK (AMPK $\alpha)$ in lipid metabolism, the effect of diosgenin on the activation of AMPK in vitro was first examined by using a specific anti-phospho-Thr-172 AMPK antibody. Under both LG and HG conditions, diosgenin treatment for $24 \mathrm{~h}$ significantly increased the phosphorylated AMPK levels in a dose-dependent manner (Fig. 1A and B). No changes in endogenous AMPK protein were observed by immunoblotting in both LG and HG medium. ACC is a downstream target of AMPK. The phosphorylation of ACC was next determined. Western blot analysis revealed that diosgenin obviously induced phosphorylation of ACC in a dose-dependent manner (Fig. 1A and B).

Therefore, it was further investigated whether diosgenin prevented HG-induced lipid accumulation in HepG2 cells. As shown in Fig. 1C, treatment with a high concentration of glucose for $24 \mathrm{~h}$ significantly increased the TG level in HepG2 cells. Diosgenin $(10,25$ and $50 \mu \mathrm{M})$ significantly inhibited HG-induced TG accumulation in HepG2 cells. High concentration of glucose also induced a significant increase of SREBP-1c mRNA (Fig. 1D), while diosgenin treatment suppressed the increase of SREBP-1c mRNA level. To further confirm the effect of diosgenin on TG accumulation, and that SREBP-1c mRNA expression is mediated by the AMPK pathway, compound C (an inhibitor of AMPK) was applied. As shown in Fig. 1E and F, the inhibition of TG and SREBP-1c mRNA in HepG2 cells by diosgenin was partially blocked by compound $\mathrm{C}$.

Diosgenin inhibits LXR $\alpha$ expression in HepG2 cells. Although activation of LXR $\alpha$ improves glucose tolerance and insulin resistance, it is also able to regulate the transcription of several lipid metabolism-related genes, including SREBP-1c, which lead to hyperlipidemia and hepatic steatosis (20-22). To further investigate whether the lipid-lowering effect of diosgenin was associated with LXR $\alpha$, the LXR $\alpha$ mRNA expression following diosgenin treatment was examined. $\mathrm{HG}$ induced a significant upregulation of LXR $\alpha$ mRNA in HepG2 cells (Fig. 2A). Treatment with diosgenin significantly lowered the expression of LXR $\alpha$ mRNA. Diosgenin also significantly inhibited LXR $\alpha$ ligand T0901317-induced LXR $\alpha$ and SREBP-1c mRNA upregulation (Fig. 2B and C), which was not abolished by compound $\mathrm{C}$.

Diosgenin decreases the body and liver weight of HFD-fed rats. There was no significant difference in the body weight among the five groups prior to the experiment (Fig. 3A). Following feeding with HFD for 16 weeks, the body weight of the rats in the model group was significantly increased compared with the control group (Fig. 3A). Diosgenin treatment significantly suppressed weight gain in HFD-fed rats. The liver weight of HFD-fed rats was also significantly increased compared with 
A

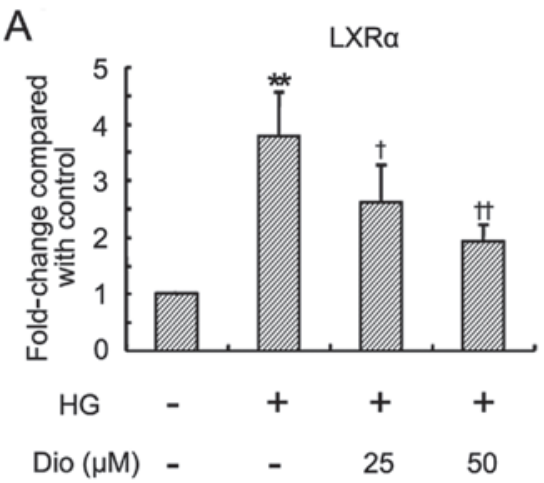

B

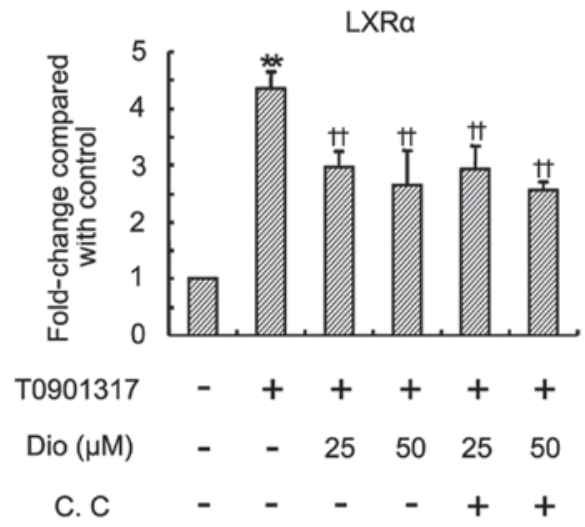

C

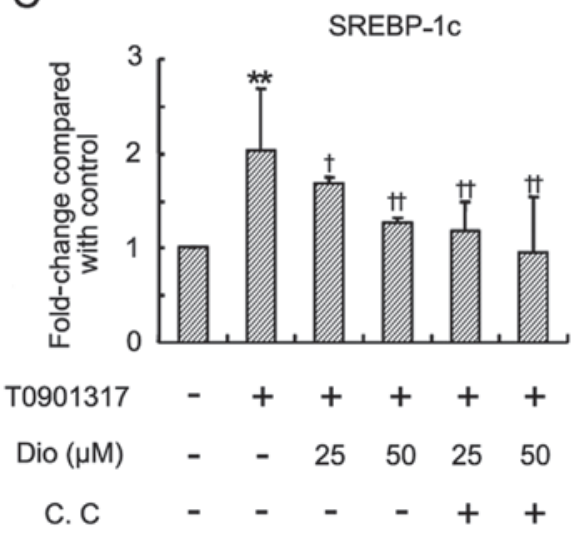

Figure 2. Diosgenin inhits liver X receptor (LXR) $\alpha$ mRNA in HepG2 cells. (A) Diosgenin inhibits high glucose (HG)-induced increase of LXR $\alpha$ mRNA in HepG2 cells; (B and C) diosgenin inhibits T0901317-induced increase of LXR $\alpha$ and sterol regulatory element-binding protein-1c (SREBP-1c) mRNA, respectively. The data are presented as mean \pm standard deviation, $n=3$. ${ }^{* *} \mathrm{P}<0.01$ compared with the control group; ${ }^{\dagger} \mathrm{P}<0.05$ and ${ }^{\dagger} \mathrm{P}<0.01$ compared with the HG group. C.C, compound C.

that of normal diet-fed rats (Fig. 3B). The liver weights of rats in the HDD and LDD groups were lower compared with those in the model group, while no significant difference was observed between the model and FEN groups.

Diosgenin ameliorates hepatic lipid accumulation in $H F D$-fed rats. The accumulation of intracellular TG in the liver parenchyma is the main pathological change observed in NAFLD (23). As shown in Fig. 4A, the Oil Red O staining revealed an obvious lipid accumulation in the liver of HFD-fed

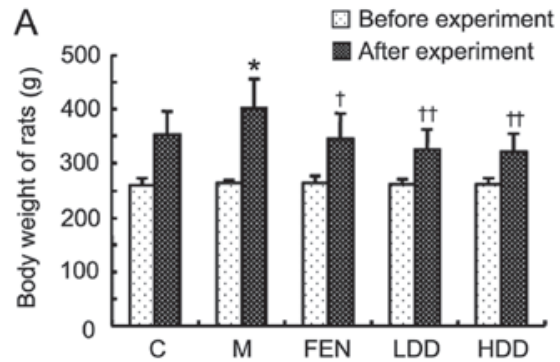

B

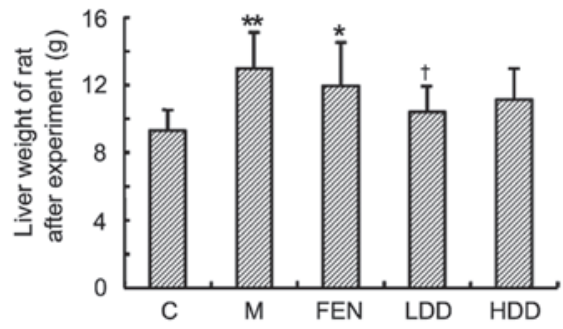

Figure 3. Diosgenin decreases the body and liver weight of high-fat diet-fed rats. (A) Body weight of rats before and after the experiment; (B) liver weight of rats after the experiment. The data are presented as mean \pm standard deviation, $\mathrm{n}=8$. ${ }^{*} \mathrm{P}<0.05$ and ${ }^{* *} \mathrm{P}<0.01$ compared with the control group; ${ }^{\dagger} \mathrm{P}<0.05$ and ${ }^{\dagger} \mathrm{P}<0.01$ compared with the model group. $\mathrm{C}$, control group; $\mathrm{M}$, model group; FEN, fenofibrate group; LDD, low-dose diosgenin group; HDD, high-dose diosgenin group.

rats compared with normal diet-fed rats. H\&E staining demonstrated that the increase in hepatic adipose infiltration in HFD-fed rats was significantly reduced by diosgenin and/or fenofibrate administration. Both diosgenin and fenofibrate treatment ameliorated the lipid deposition in the rat liver. The hepatic TG content in HFD-fed rats was higher compared with that in normal diet-fed rats (Fig. 4B). Diosgenin and fenofibrate also significantly reduced the TG content in the rat liver.

Diosgenin also significantly decreased the serum TG levels (Fig. 4C), while it had little effect on the serum TC levels (Fig. 4D). The high-density lipoprotein (HDL) and low-density lipoprotein cholesterol were both significantly increased in HFD-fed rats (Fig. 4E and F). The HDL cholesterol levels in the diosgenin groups were higher compared with those in the model group. However, the differences between the diosgenin and model groups were not significant.

Diosgenin ameliorates hepatic dysfunction in HFD-fed rats. The ALT level in HFD-fed rats was significantly increased compared with that in normal diet-fed rats (Fig. 5A). Diosgenin treatment significantly reduced the ALT levels, whereas fenofibrate further elevated the level of ALT. The AST level did not significantly increase in the HFD-fed rats (Fig. 5B). There was no significant difference in the AST levels between the diosgenin treatment groups and the model group, while fenofibrate treatment significantly increased the AST level.

Diosgenin increases AMPK and ACC phosphorylation and suppresses LXR in HFD-fed rats liver. Subsequently, the levels of AMPK, ACC and LXR $\alpha$ in the livers of HFD-fed rats were further investigated by western blot analysis. As shown in Fig. 6A-C, the levels of p-AMPK and p-ACC were significantly increased in the diosgenin- and fenofibrate-treated 
A

A

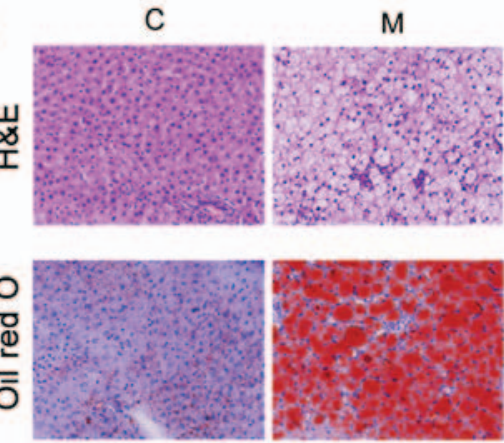

C

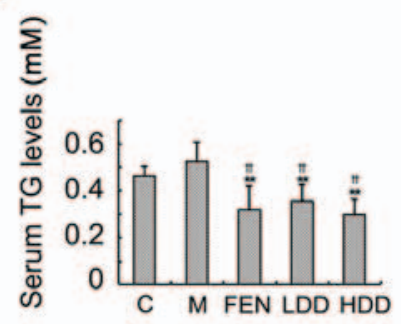

D

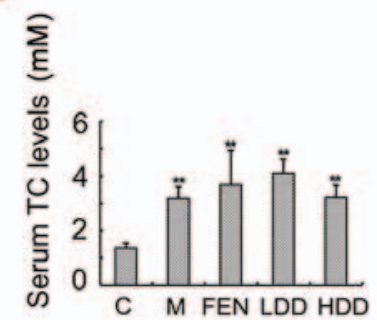

LDD
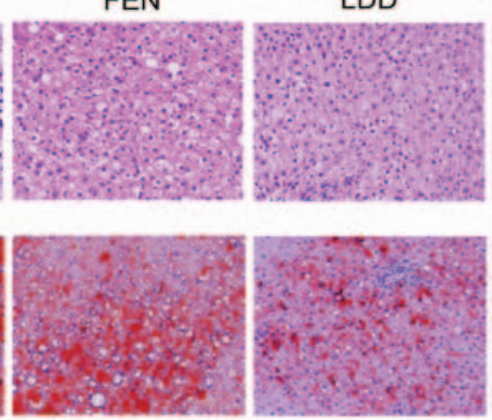

E

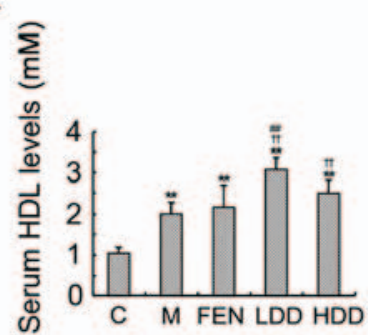

B

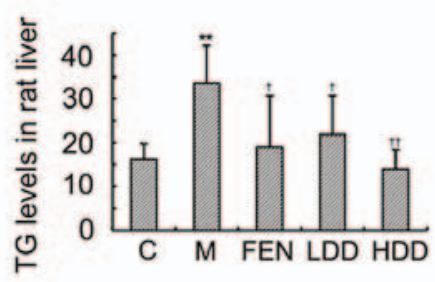

F

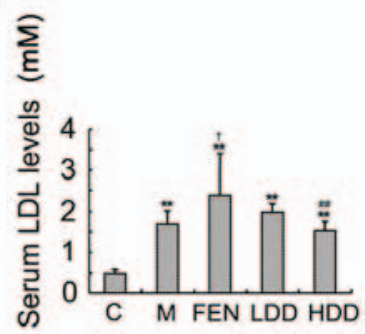

Figure 4. Diosgenin ameliorates the hepatic lipid accumulation in high-fat diet-fed rats. (A) Hematoxylin and eosin (H\&E) and Oil red O staining of the rat liver; (B) hepatic triglyceride (TG) levels; (C-F) serum TG, total cholesterol (TC), high-density lipoprotein (HDL) and low-density lipoprotein (LDL) cholesterol levels of rats. The data are presented as mean \pm standard deviation, $n=8$. ${ }^{*} \mathrm{P}<0.05$ and ${ }^{* *} \mathrm{P}<0.01$ compared with the control group; ${ }^{\dagger} \mathrm{P}<0.05$ and ${ }^{+\dagger} \mathrm{P}<0.01$ compared with the model group; ${ }^{\#} \mathrm{P}<0.01$ compared with the fenofibrate group. $\mathrm{C}$, control group; M, model group; FEN, fenofibrate group; LDD, low-dose diosgenin group; HDD, high-dose diosgenin group.
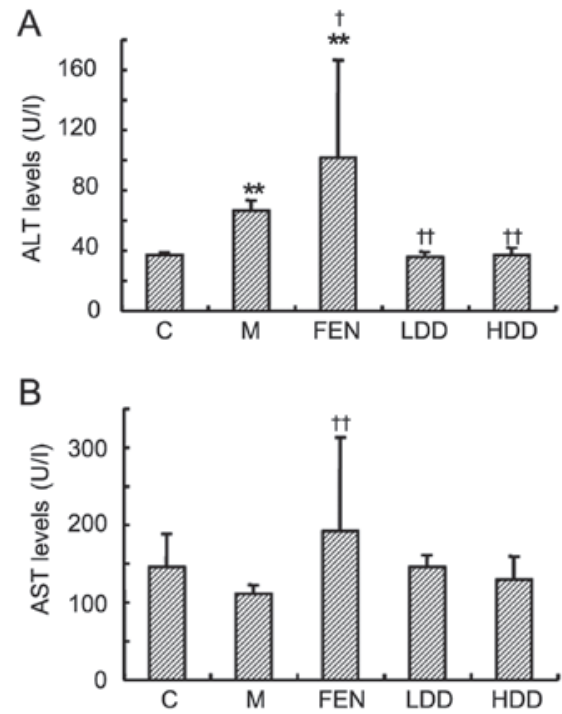

Figure 5. Alanine aminotransferase (ALT) and aspartate aminotransferase (AST) levels of the rats after the experiment. (A and B) ALT and AST levels of the rats, respectively. The data are presented as mean \pm standard deviation, $\mathrm{n}=8$. ${ }^{* *} \mathrm{P}<0.01$ compared with the control group; ${ }^{\dagger} \mathrm{P}<0.05$ and ${ }^{\dagger} \mathrm{P}<0.01$ compared with the model group. $\mathrm{C}$, control group; $\mathrm{M}$, model group; FEN, fenofibrate group; LDD, low-dose diosgenin group; HDD, high-dose diosgenin group.

groups compared with the model group. However, there were no significant differences in the total AMPK and ACC between the diosgenin- and fenofibrate-treated groups and the model group. Diosgenin treatment significantly suppressed the LXR $\alpha$ level compared with the model group (Fig. 6D). However, fenofibrate treatment further upregulated the LXR $\alpha$ level compared with the model group.

\section{Discussion}

NAFLD is characterized by increased hepatocellular lipid accumulation and is frequently associated with steatohepatitis and liver injury, which may eventually result in severe liver damage, including hepatic fibrosis and cirrhosis, or even liver cancer. The data of the present study demonstrated that diosgenin was able to activate AMPK, thereby inhibiting lipid accumulation in hepatocytes. Furthermore, the effect of diosgenin on lipid accumulation was abolished by compound $\mathrm{C}$, an inhibitor of AMPK, suggesting that the effect of diosgenin on NAFLD is AMPK-dependent. Moreover, our results indicated that diosgenin may also inhibit $\mathrm{LXR} \alpha$ and $\operatorname{LXR} \alpha$ agonist-induced SREBP-1c upregulation independently of AMPK. In addition, diosgenin also alleviated the HFD-induced liver function disruption in rats, adding an advantage over the traditional lipid-lowering medicines.

High glucose concentration is associated with insulin resistance, which leads to elevated hepatic glucose production, hyperglycemia and hyperlipidemia (24). Exposure of HepG2 cells to high concentrations of glucose inhibits the phosphorylation of AMPK and ACC (25). It has been demonstrated that the inhibition of AMPK and ACC phosphorylation contributes to extensive glucose-induced lipid accumulation in HepG2 cells (14). By contrast, metformin, an AMPK activator, increases phosphorylation of ACC and effectively attenuates lipid accumulation in HepG2 cells induced by high concentration of glucose (14). In the present study, it was first demonstrated that diosgenin increased the phosphorylation of AMPK and ACC under conditions of low and high concentrations of glucose, indicating that diosgenin may be capable of regulating lipid metabolism in the liver. Therefore, a model 
A

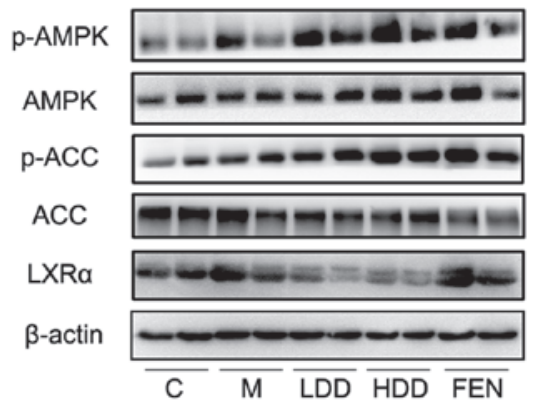

C

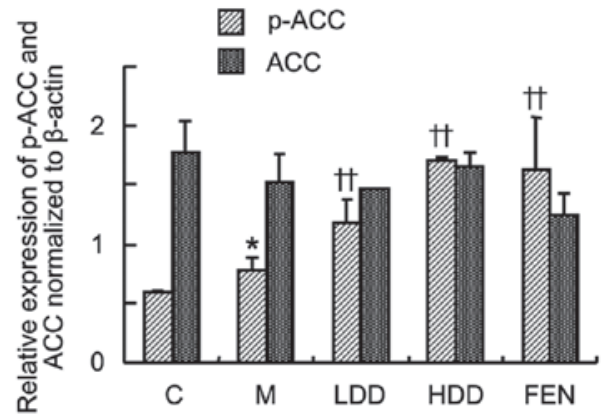

B

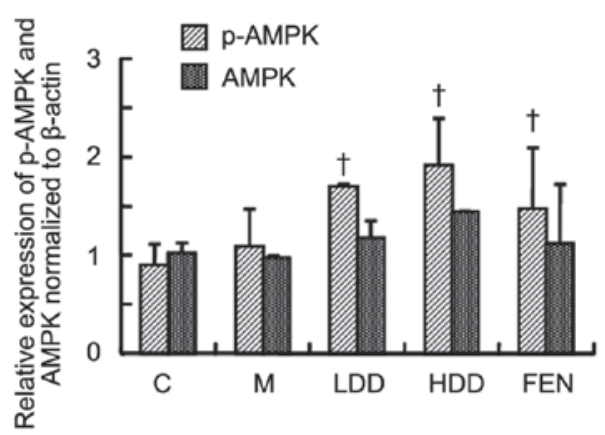

D

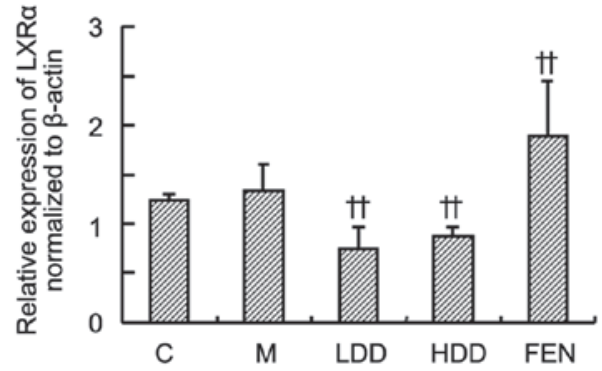

Figure 6. Diosgenin increases AMP-activated protein kinase (AMPK) and acetyl-CoA carboxylase (ACC) phosphorylation and suppresses liver X receptor (LXR) $\alpha$ in the liver of high-fat diet (HFD)-fed rats. (A) Western blot analysis of p-AMPK, p-ACC and LXR $\alpha$ in the liver of HFD-fed rats; (B-D) relative levels of p-AMPK/AMPK, p-ACC/ACC and LXR $\alpha$ normalized to $\beta$-actin, respectively. The data are presented as mean \pm standard deviation, $\mathrm{n}=8$. * $<<0.05$ compared with the control group; ${ }^{\dagger} \mathrm{P}<0.05$ and ${ }^{\dagger} \mathrm{P}<0.01$ compared with the model group. $\mathrm{C}$, control group; M, model group; FEN, fenofibrate group; LDD, low-dose diosgenin group; HDD, high-dose diosgenin group.

of insulin resistance induced by high concentration of glucose was used to test the effect of diosgenin on hepatic TG accumulation. As expected, diosgenin treatment decreased the TG level in HG-treated HepG2 cells. Moreover, the effects of diosgenin on lipid accumulation were abolished by the inhibitor of AMPK. These data suggest that diosgenin may be promising for preventing HG-induced lipid accumulation in the liver through activating the AMPK pathway.

SREBP1 is the most important transcription factor regulating lipogenesis in the liver, and is primarily responsible for the regulation of genes involved in fatty acid biosynthesis, such as FAS. AMPK suppresses SREBP-1c cleavage and nuclear translocation, and represses SREBP-1c target gene expression in hepatocytes exposed to $\mathrm{HG}$, leading to reduced lipogenesis and lipid accumulation (26). In the present study, diosgenin suppressed HG-induced SREBP-1c mRNA upregulation, which was partially blocked by the AMPK inhibitor, suggesting that other pathways may also participate in the lipid-lowering effect of diosgenin. SREBP1 is also a major target of LXR $\alpha$, which upregulates lipogenesis (27). The present study demonstrated that the HG-induced increase of LXR $\alpha$ mRNA is also suppressed by diosgenin. Lee et al reported that AMPK activation suppresses LXR $\alpha$ agonist-induced SREBP-1c mRNA (28). The present study demonstrated that LXR $\alpha$ agonist increases the expression of LXR $\alpha$ and SREBP-1c mRNA, which was not blocked by the AMPK inhibitor. These results suggest that the inhibition of LXR $\alpha /$ SREBP-1c signaling pathway by diosgenin is independent of AMPK.
HFD is one of the most important risk factors associated with the incidence of NAFLD $(29,30)$. Rodents fed a HFD exhibit visceral adiposity, hyperglycemia, dyslipidemia, hyperinsulinemia and hepatic steatosis, which are findings similar to those in human NAFLD (31). In order to investigate the in vivo effect of diosgenin on NAFLD, a rat model fed with HFD was used. In accordance with previous studies, HFD caused obvious increases in rat body and liver weight, which were significantly decreased by diosgenin. As determined by Oil red $\mathrm{O}$ and H\&E staining, diosgenin obviously ameliorated lipid accumulation in the liver. This result was confirmed by quantification of liver TG content. Considering the improvement of lipid accumulation, it is obvious that diosgenin treatment was able to alleviate NAFLD-induced liver injury.

ALT and AST are common indicators of liver injury in the majority of liver diseases (32). Under normal conditions, ALT and AST are mainly localized in the cytoplasm of hepatocytes. When the hepatocytes are damaged, these enzymes are released from the cells into the circulation, leading to elevated hepatic and serum ALT and/or AST levels (33). In the present study, the elevated serum ALT level in NAFLD rats was reduced by diosgenin administration. However, fenofibrate treatment significantly increased the serum ALT and AST levels. Our results indicate an advantage of diosgenin over fenofibrate in terms of liver function in NAFLD.

In conclusion, the present study demonstrated that diosgenin was able to activate the AMPK pathway and suppress LXR $\alpha$, thereby ameliorating the hepatic lipid accumulation 
in vitro and in vivo. Diosgenin also improved the HFD-induced liver function disturbance. These data suggest that diosgenin is a potential agent for preventing NAFLD through the AMPK and LXR $\alpha$ pathways.

\section{Acknowledgements}

The present study was supported by grants from the National Natural Science Foundation of China (nos. 81430101, 81673655 and 81603458) and the E-Institutes of Shanghai Municipal Education Commission (no. E03008).

\section{References}

1. Caballería L, Auladell MA, Torán P, Miranda D, Aznar J, Pera G, Gil D, Muñoz L, Planas J, Canut S, et al: Prevalence and factors associated with the presence of non alcoholic fatty liver disease in an apparently healthy adult population in primary care units. BMC Gastroenterol 7: 41, 2007.

2. Hu X, Huang Y, Bao Z, Wang Y, Shi D, Liu F, Gao Z and Yu X: Prevalence and factors associated with nonalcoholic fatty liver disease in Shanghai work-units. BMC Gastroenterol 12: 123, 2012.

3. Welsh JA, Karpen S and Vos MB: Increasing prevalence of nonalcoholic fatty liver disease among United States adolescents, 1988-1994 to 2007-2010. J Pediatr 162: 496-500, 2013.

4. Margini C and Dufour JF: The story of HCC in NAFLD: from epidemiology, across pathogenesis, to prevention and treatment. Liver Int 36: 317-324, 2016.

5. Kahn BB, Alquier T, Carling D and Hardie DG: AMP-activated protein kinase: ancient energy gauge provides clues to modern understanding of metabolism. Cell Metab 1: 15-25, 2005.

6. Hardie DG: AMP-activated/SNF1 protein kinases: conserved guardians of cellular energy. Nat Rev Mol Cell Biol 8: 774-785, 2007

7. Santamarina AB, Oliveira JL, Silva FP, Carnier J, Mennitti LV, Santana AA, de Souza GH, Ribeiro EB, Oller do Nascimento CM, Lira FS, et al: Green tea extract rich in epigallocatechin-3-gallate prevents fatty liver by AMPK activation via LKB1 in mice fed a high-fat diet. PLoS One 10: e0141227, 2015.

8. Li H, Min Q, Ouyang C, Lee J, He C, Zou MH and Xie Z: AMPK activation prevents excess nutrient-induced hepatic lipid accumulation by inhibiting mTORC1 signaling and endoplasmic reticulum stress response. Biochim Biophys Acta 1842: 1844-1854, 2014

9. Yang Y, Li W, Liu Y, Sun Y, Li Y, Yao Q, Li J, Zhang Q, Gao Y, Gao L, et al: Alpha-lipoic acid improves high-fat diet-induced hepatic steatosis by modulating the transcription factors SREBP-1, FoxO1 and Nrf2 via the SIRT1/LKB1/AMPK pathway. J Nutr Biochem 25: 1207-1217, 2014.

10. Yuan $\mathrm{H}$, Weng $\mathrm{C}$, Yang Y, Huang L and Xing X: Resistin, an adipokine, may affect the improvement of insulin sensitivity in the metabolic syndrome patient treated with metformin. Med Hypotheses 81: 969-971, 2013.

11. de Mello VD, Kolehmainen M, Pulkkinen L, Schwab U, Mager U, Laaksonen DE, Niskanen L, Gylling H, Atalay M, Rauramaa $\mathrm{R}$, et al: Downregulation of genes involved in NFkappaB activation in peripheral blood mononuclear cells after weight loss is associated with the improvement of insulin sensitivity in individuals with the metabolic syndrome: The GENOBIN study. Diabetologia 51: 2060-2067, 2008.

12. Xu X, Huang P, Yang B, Wang X and Xia J: Roles of CXCL5 on migration and invasion of liver cancer cells. J Transl Med 12: 193, 2014.

13. Chen Y, Xu X, Zhang Y, Liu K, Huang F, Liu B and Kou J: Diosgenin regulates adipokine expression in perivascular adipose tissue and ameliorates endothelial dysfunction via regulation of AMPK. J Steroid Biochem Mol Biol 155: 155-165, 2016.

14. Zang M, Zuccollo A, Hou X, Nagata D, Walsh K, Herscovitz H, Brecher P, Ruderman NB and Cohen RA: AMP-activated protein kinase is required for the lipid-lowering effect of metformin in insulin-resistant human HepG2 cells. J Biol Chem 279: 47898-47905, 2004.
15. Uemura T, Goto T, Kang MS, Mizoguchi N, Hirai S, Lee JY, Nakano Y, Shono J, Hoshino S, Taketani K, et al: Diosgenin, the main aglycon of fenugreek, inhibits LXR $\alpha$ activity in HepG2 cells and decreases plasma and hepatic triglycerides in obese diabetic mice. J Nutr 141: 17-23, 2011.

16. Ghosh S, Sikdar S, Mukherjee A and Khuda-Bukhsh AR: Evaluation of chemopreventive potentials of ethanolic extract of Ruta graveolens against A375 skin melanoma cells in vitro and induced skin cancer in mice in vivo. J Integr Med 13: 34-44, 2015

17. Wu J, Zhang H, Zheng $\mathrm{H}$ and Jiang Y: Hepatic inflammation scores correlate with common carotid intima-media thickness in rats with NAFLD induced by a high-fat diet. BMC Vet Res 10: 162,2014

18. Akaslan SB, Degertekin CK, Yilmaz G, Cakir N, Arslan M and Toruner FB: Effects of sitagliptin on nonalcoholic fatty liver disease in diet-induced obese rats. Metab Syndr Relat Disord 11: 243-250, 2013.

19. Park KG, Min AK, Koh EH, Kim HS, Kim MO, Park HS, Kim YD, Yoon TS, Jang BK, Hwang JS, et al: Alpha-lipoic acid decreases hepatic lipogenesis through adenosine monophosphate-activated protein kinase (AMPK)-dependent and AMPK-independent pathways. Hepatology 48: 1477-1486, 2008.

20. Edwards PA, Kast HR and Anisfeld AM: BAREing it all: the adoption of LXR and FXR and their roles in lipid homeostasis. J Lipid Res 43: 2-12, 2002.

21. Gao M and Liu D: The liver X receptor agonist T0901317 protects mice from high fat diet-induced obesity and insulin resistance. AAPS J 15: 258-266, 2013.

22. Chisholm JW, Hong J, Mills SA and Lawn RM: The LXR ligand T0901317 induces severe lipogenesis in the $\mathrm{db} / \mathrm{db}$ diabetic mouse. J Lipid Res 44: 2039-2048, 2003.

23. Jegatheesan P, Beutheu S, Freese K, Waligora-Dupriet AJ, Nubret E, Butel MJ, Bergheim I and De Bandt JP: Preventive effects of citrulline on Western diet-induced non-alcoholic fatty liver disease in rats. Br J Nutr 116: 191-203, 2016.

24. Tsujimoto S, Kishina M, Koda M, Yamamoto Y, Tanaka K, Harada Y, Yoshida A and Hisatome I: Nimesulide, a cyclooxygenase-2 selective inhibitor, suppresses obesity-related non-alcoholic fatty liver disease and hepatic insulin resistance through the regulation of peroxisome proliferator-activated receptor $\gamma$. Int J Mol Med 38: 721-728, 2016.

25. Dyck JR, Kudo N, Barr AJ, Davies SP, Hardie DG and Lopaschuk GD: Phosphorylation control of cardiac acetyl-CoA carboxylase by cAMP-dependent protein kinase and 5'-AMP activated protein kinase. Eur J Biochem 262: 184-190, 1999.

26. Li Y, Xu S, Mihaylova MM, Zheng B, Hou X, Jiang B, Park O, Luo Z, Lefai E, Shyy JY, et al: AMPK phosphorylates and inhibits SREBP activity to attenuate hepatic steatosis and atherosclerosis in diet-induced insulin-resistant mice. Cell Metab 13: 376-388, 2011.

27. Schultz JR, Tu H, Luk A, Repa JJ, Medina JC, Li L, Schwendner S, Wang S, Thoolen M, Mangelsdorf DJ, et al: Role of LXRs in control of lipogenesis. Genes Dev 14: 2831-2838, 2000.

28. Lee J, Hong SW, Park SE, Rhee EJ, Park CY, Oh KW, Park SW and Lee WY: AMP-activated protein kinase suppresses the expression of LXR/SREBP-1 signaling-induced ANGPTL8 in HepG2 cells. Mol Cell Endocrinol 414: 148-155, 2015.

29. Cabrera D, Ruiz A, Cabello-Verrugio C, Brandan E, Estrada L, Pizarro M, Solis N, Torres J, Barrera F and Arrese M: Diet-induced nonalcoholic fatty liver disease is associated with sarcopenia and decreased serum insulin-like growth factor-1. Dig Dis Sci 61: 3190-3198, 2016.

30. Wang C, Tao Q, Wang X, Wang X and Zhang X: Impact of high-fat diet on liver genes expression profiles in mice model of nonalcoholic fatty liver disease. Environ Toxicol Pharmacol 45: 52-62, 2016.

31. Xu ZJ, Fan JG, Ding XD, Qiao L and Wang GL: Characterization of high-fat, diet-induced, non-alcoholic steatohepatitis with fibrosis in rats. Dig Dis Sci 55: 931-940, 2010.

32. Jiang SL, Hu XD and Liu P: Immunomodulation and liver protection of Yinchenhao decoction against concanavalin A-induced chronic liver injury in mice. J Integr Med 13: 262-268, 2015

33. Moghadam AR, Tutunchi S, Namvaran-Abbas-Abad A, Yazdi M, Bonyadi F, Mohajeri D, Mazani M, Marzban H, Łos MJ and Ghavami S: Pre-administration of turmeric prevents methotrexate-induced liver toxicity and oxidative stress. BMC Complement Altern Med 15: 246, 2015. 